\title{
La problematización: Herramienta para facilitar el aprendizaje auténtico de las ciencias en el nivel elemental
}

Enviado: 2 de octubre de 2020 / Aceptado: 19 de octubre de 2020

Publicado: 30 de diciembre de 2020

LIZZETTE M. VELÁZQUEZ RIVERA

Departamento de Programas y Enseñanza, Facultad de Educación Universidad de Puerto Rico, Puerto Rico

lizzette.velazquez1@upr.edu

LINDA CLARK MORA

Escuela Elemental de la Universidad de PR, Facultad de Educación, Universidad de Puerto Rico, Puerto Rico

linda.clark@upr.edu

ISARIS R. QUIÑONES PÉREZ

San Juan, Puerto Rico

isaris.quinones@upr.edu

\section{RESUMEN}

En este artículo se presenta cómo la problematización, que se facilitó por medio del Proyecto Réplicas para la Vida, sirvió de contexto para que estudiantes de sexto grado aprendieran de manera auténtica acerca de ecología. Se describe cómo a través de diferentes actividades realizadas durante un semestre, dentro y fuera del salón de clases, los estudiantes experimentaron un aprendizaje auténtico que les ayudó a tomar conciencia de su impacto ambiental y su rol en la sociedad. Las actividades medulares del proyecto se dividieron en: a) actividades para facilitar la problematización, b) actividades de aprendizaje y c) actividades para aplicar lo aprendido. A través de este

\section{ABSTRACT}

Problematization: A tool to facilitate authentic science learning at the elementary level

This article presents how the problematization, which was facilitated through the Life's Replica Project, served as a context for sixth grade students to learn about ecology in an authentic way. It describes how through different activities conducted during one semester, in and out of the classroom, students experienced authentic learning that helped them become aware of their environmental impact and their role in society. The core activities of the project were divided into: a) activities to facilitate problematiza- 
proyecto los estudiantes investigaron un problema, desarrollaron un producto y prestaron un servicio a su comunidad. Finalmente, se presentan los logros más importantes del proyecto. Se concluye que la problematización es una herramienta útil para facilitar el aprendizaje auténtico de las ciencias en el nivel elemental.

Palabras Clave: aprendizaje auténtico; aprendizaje basado en problemas, aprendizaje basado en proyectos, aprendizaje en servicio, ciencias de escuela elemental, ecología. tion, b) learning activities, and c) activities to apply what was learned. Through this project, students investigated a problem, developed a product, and provided a service to their community. Finally, the most important achievements of the project are presented. In conclusion, the problematization is a useful tool to facilitate authentic science learning at the elementary level.

Keywords: authentic learning, problem based learning, project based learning, service learning, elementary school science, ecology.

\section{INTRODUCCIÓN}

Puerto Rico se distingue por sus hermosas playas. Por tal razón, el paisaje de las playas para un puertorriqueño es algo muy familiar. Sin embargo, no es común ver la Playa de Buyé en Cabo Rojo, una playa muy hermosa y popular en la costa suroeste de la isla, invadida un viernes por 32 estudiantes de sexto grado y 16 futuros maestros de ciencias. Agrupados en estaciones de trabajo, los estudiantes tuvieron la oportunidad de desempeñar el rol de ecoingenieros embajadores de la sustentabilidad, integrando las disciplinas STEM (Science, Technology, Engineering and Mathematics) y el diseño de ingeniería en la búsqueda de soluciones a problemas causados por el impacto humano en el Planeta Tierra.

La experiencia de aprendizaje formó parte de un Residencial Educativo donde los estudiantes tuvieron que pernoctar de jueves a viernes en un lugar cercano a uno de los ecosistemas de la zona suroeste de Puerto Rico. Durante la tarde del jueves participaron en una Sesión Legislativa de la Asamblea Municipal de Cabo Rojo. Los estudiantes observaron cómo se presentaron y defendieron los proyectos de sustentabilidad ambiental en el gobierno permitiéndoles tener mejor entendimiento de los asuntos ambientales que afectan a las sociedades contemporáneas. De esta manera, aprendieron acerca de lo que hace un embajador de la sustentabilidad. Para los estudiantes este residencial fue una experiencia muy significativa:

\footnotetext{
"Esta experiencia me ayudó a tener acceso a diversos recursos naturales y a aprender cómo defenderlos y cuidarlos para que otras generaciones las puedan disfrutar. Además, asumí mi rol en la asamblea educándome sobre las leyes que benefician nuestros recursos del país.”
} 
Aunque los viajes de campo no son nada nuevo en la educación, la pregunta que debemos hacernos es ¿cómo trascender la emoción del viaje para facilitar aprendizajes auténticos en las ciencias? Este Residencial Educativo fue sólo una de las actividades medulares del Proyecto Réplicas para la vida: fóvenes conscientes de su impacto ambiental y su rol en la sociedad, el cual duró un semestre escolar. El mismo sirvió de contexto para que los estudiantes de sexto grado aprendieran en torno al impacto humano, los ecosistemas y la necesidad de su conservación.

\section{2. ¿CÓMO FACILITAR EL APRENDIZAJE AUTÉNTICO?}

En muchas ocasiones, entender o saber de algo consiste en pensar de la manera que nos han enseñado y hemos aprendido. Usualmente no tenemos problema al utilizar esa forma de pensar o de aprender. Sin embargo, cuando una perturbación opera en contra de ese modo de pensar, se crea un conflicto cognitivo que debemos resolver. Ese conflicto cognitivo entre lo que sabemos y lo que ocurre o se nos presenta, genera una necesidad por aprender (Piaget, 1980). Esto es, que el aprendizaje se produce de forma natural cuando tenemos la necesidad de aprender provocado por un conflicto cognitivo, y éste se resuelve, al interaccionar por medio de una actividad intelectual con personas, objetos o fenómenos (Figarella García, 2018).

Los educadores, deben provocar conflictos cognitivos en sus estudiantes, de manera que se genere en ellos la necesidad de reorganizar las ideas, pensar de manera diferente y aprender. El aprendizaje debe involucrar todas las habilidades cognitivas, motrices, emocionales y afectivas. Para ello, el estudiante debe sentir que lo que aprende es importante porque lo necesita. En otras palabras, que es significativo. El aprendizaje significativo surge primordialmente de la necesidad de aprender. Ocurre cuando lo que se aprende sirve, se utiliza y es valorado por el estudiante como algo primordial (Velázquez Rivera \& Figarella García, 2018). Más aún, el estudiante reconoce la funcionalidad de dicho aprendizaje y puede utilizarlo inmediatamente en su vida.

Para que el aprendizaje sea significativo, es necesario que las actividades sean apropiadas, relevantes para el estudiante y que le permita relacionar el conocimiento previo con lo nuevo; esto es, que sea auténtico. El aprendizaje auténtico ocurre cuando el estudiante experimenta la realidad, contrario a aprender simplemente hechos en situaciones abstractas y artificiales (Newmann et al.,1995). Fundamentado en el enfoque constructivista, en el que el aprendizaje es un proceso activo, los educadores brindan oportunidades a los estudiantes para construir su conocimiento centrado en la solución de problemas y en el pensamiento crítico del mundo real. El currículo auténtico logra que las actividades sean relevantes para el estudiante de ma- 
nera que despierte su necesidad de aprender y que facilite cambios de estado, cambios en su manera de pensar, sentir y actuar (Velázquez Rivera \& Figarella García, 2018).

Para Velázquez Rivera y Figarella García (2018), la creación de currículo auténtico se promueve por medio de la problematización en el aprendizaje. La problematización se refiere al proceso de facilitar la creación de conflictos cognitivos en los estudiantes, de manera que los mueva a analizar, reflexionar, investigar, crear, actuar y evaluar para construir nuevos aprendizajes. La problematización en el aprendizaje favorece que este sea auténtico y para toda la vida, ya que invita de forma natural a los estudiantes a querer aprender pues les provoca un conflicto cognitivo que estimula su necesidad de conocimiento. La vida diaria está llena de retos, de problemas por resolver, de proyectos que hacer y de servicios a prestar que pueden servir de contexto para facilitar la creación de un currículo auténtico (Velázquez Rivera, Figarella García \& Clark Mora, 2016). Precisamente, el Proyecto Réplicas para la vida se desarrolló centrado en la problematización al utilizar un problema real y la necesidad de concienciar a otros en torno al mismo (servicio comunitario) para despertar la urgencia por aprender de manera auténtica.

\section{DESCRIPCIÓN DEL PROYECTO RÉPLICAS PARA LA VIDA}

La vida como la conocemos solo existe en la Tierra. Por lo tanto, no hay duda de la dependencia que tenemos de los recursos de la Tierra para la vida, así como del impacto que esta vida tiene en ellos, sobre todo del impacto humano. Entonces, nos preguntamos ¿Qué pudiera hacer la humanidad si la vida en la Tierra ya no fuera sostenible debido al impacto que hemos causado al planeta y a lo limitado de sus recursos?

Entre las opciones que se han investigado, están las de replicar, en lugares fuera y dentro de la Tierra, las condiciones que permiten la vida. Por ejemplo, fuera del planeta hemos creado la estación espacial, y dentro de él hemos creado unas biosferas artificiales más o menos exitosas. Aun así, para diversos científicos, organizaciones y agencias es sumamente importante conocer, valorar y proteger esas condiciones que permiten la vida. Por ejemplo, la Asociación de las Naciones Unidas impulsó la iniciativa de los Objetivos de Desarrollo Sostenible (ODS) para transformar nuestro mundo en uno sostenible para todos (Naciones Unidas, 2020). Por tal razón, ellos necesitan del apoyo de todos los terrícolas para poder lograr estos objetivos.

El Capítulo de Puerto Rico de la Asociación de las Naciones Unidas de los Estados Unidos de América (UNA-USA PR), como parte de este proyecto, solicitó apoyo a estudiantes de sexto grado de una escuela laboratorio del nivel elemental y a futuros maestros de ciencias, 
ambos de una universidad de Puerto Rico. Específicamente, a los estudiantes de sexto grado (énfasis de este artículo) se les requirió desempeñarse como eco-ingenieros embajadores de la sustentabilidad para estudiar las características e interacciones entre los factores bióticos y abióticos de ecosistemas estuarinos, de agua dulce, marinos, y terrestres (bosques) del noreste y suroeste de Puerto Rico. Además, se les solicitó que evaluaran el impacto humano en los ecosistemas estudiados y presentaran recomendaciones para atender los ODS.

En especial, se les invitó a los estudiantes a enfocarse en atender el problema y responder la siguiente pregunta-problema: ¿Cuáles interacciones y características de diferentes ecosistemas (con énfasis en los de PR) debemos replicar para propiciar las condiciones necesarias para la vida en lugares no aptos para ella? Se fundamentó en el supuesto de que la identificación de estas condiciones necesarias para la vida promoverá que los estudiantes valoren los ecosistemas y por ende se comprometan con su conservación.

\section{4. ¿CÓMO SE FACILITÓ EL APRENDIZAJE AUTÉNTICO EN EL PROYECTO?}

Uno de los elementos que facilitó el aprendizaje auténtico fue la estrategia utilizada para problematizar la Unidad de Ecosistemas. La estrategia para desarrollar las actividades de aprendizaje de la unidad de ecosistemas fue la recomendada por Velázquez Rivera y Figarella García (2018) la cual consiste en la combinación de Aprendizaje basado en problemas, Aprendizaje basado en proyectos y Aprendizaje basado en servicio. De esa forma, los estudiantes investigaron un problema, desarrollaron un producto y prestaron un servicio a la comunidad, todo como parte del Proyecto Réplicas para la Vida. Además de la estrategia Aprendizaje basado en Problema, Proyecto y Servicio, las actividades medulares desarrolladas como parte del proyecto, sirvieron a su vez, para facilitar el aprendizaje auténtico de los estudiantes de sexto grado y los futuros maestros de ciencias. Las actividades medulares del proyecto fueron categorizadas en actividades para: a) facilitar la problematización, b) aprender (la mayoría realizadas a base de lo solicitado por los estudiantes como necesario para desempeñar su rol) y c) aplicar lo aprendido. Durante el semestre escolar, las actividades se llevaron a cabo de la siguiente manera:

- Actividades para facilitar la problematización: Mediados y finales de agosto

- Actividades para aprender: Inicios de septiembre a finales de noviembre

- Actividades para aplicar lo aprendido: Inicios de diciembre 


\subsection{Actividades para facilitar la problematización}

Velázquez Rivera y Figarella García $(2016,2018)$ establecen una estructura para desarrollar el currículo auténtico centrado en la problematización en el aprendizaje. Esta estructura consiste de siete pasos que surgen de la integración de elementos comunes de las estrategias de Aprendizaje Basado en Problema, Proyecto y Servicio (ver Figura 1).

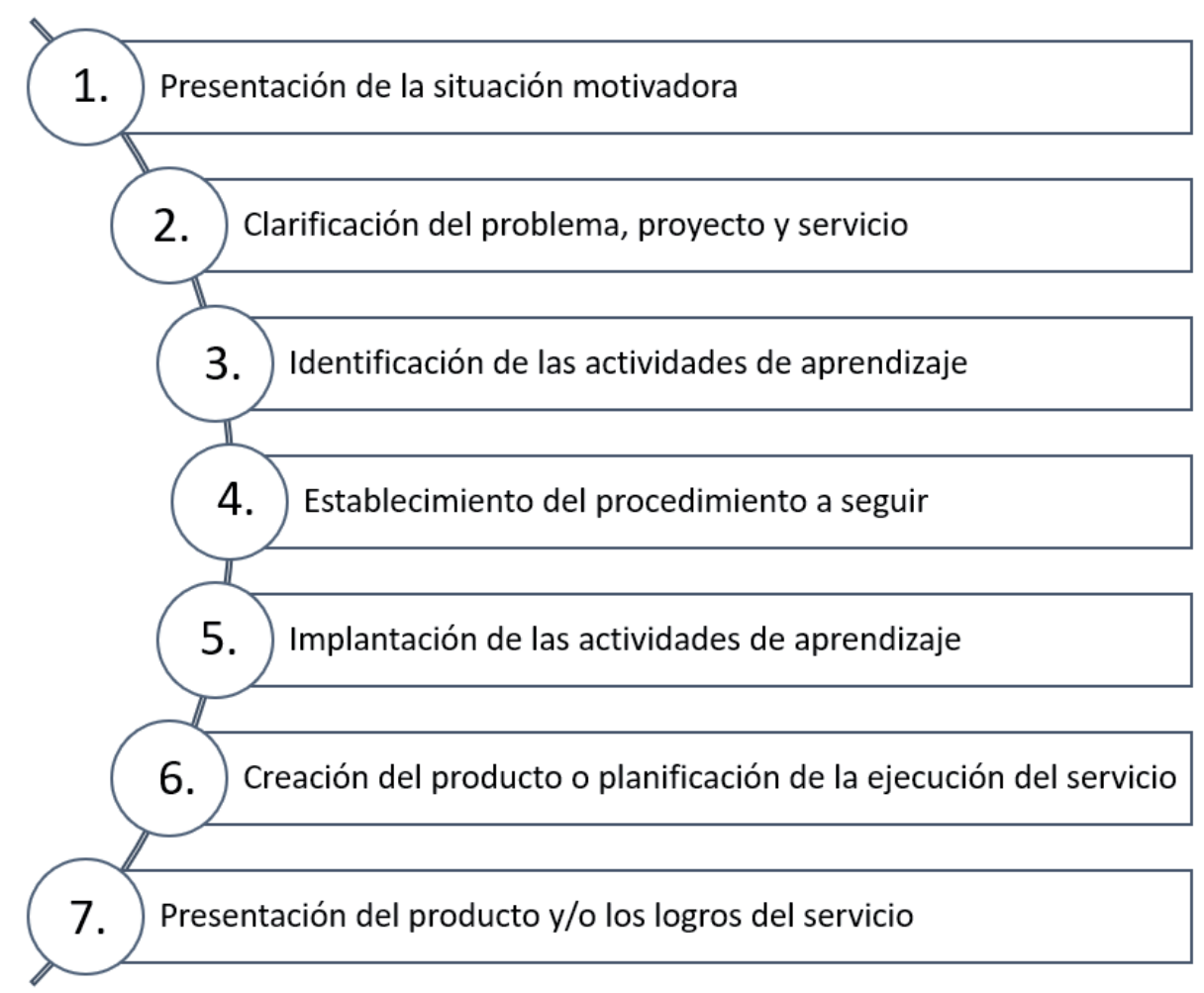

Figura 1. Pasos para facilitar la problematización en el aprendizaje

\subsubsection{Presentación de la situación motivadora}

A base de esa estructura, la problematización en el aprendizaje se inicia con la presentación de la situación motivadora en la que se le presenta al estudiante el proyecto a desarrollarse, la necesidad de estudiar un problema y de prestar un servicio comunitario. Como parte de la situación motivadora, alguien externo a la comunidad escolar, experto en el tema bajo estudio, les solicita a los estudiantes su colaboración. La misma conlleva desempeñar un rol para estudiar un problema real, presentar posibles soluciones al mismo e implantar algunas de ellas (proyecto y servicio). La presentación de la situación motivadora fue la que generó inicialmente en los estudiantes la creación de conflictos cognitivos y la necesidad de aprender 
de ecología. De esta manera, se llevó a cabo el primer paso de un currículo auténtico centrado en la problematización en el aprendizaje.

El proyecto comenzó con una presentación digital para potenciar el pensamiento crítico y facilitar el desarrollo de conflictos cognitivos, mostrándoles la necesidad de asumir el rol de eco-ingenieros embajadores de la sustentabilidad y de colaborar con la UNA-USA PR en la investigación de un problema. Los recortes de noticias, fotos y vídeos, usados en la presentación, así como el compromiso de atender los ODS, facilitaron contextualizar y dar pertinencia al tema de ecología. A su vez, provocó discusión y argumentación entre los estudiantes acerca de la importancia de conservar el ambiente frente al impacto que el ser humano ha causado a los recursos del planeta. Entre otros aspectos, los estudiantes plantearon sus preocupaciones acerca del cambio climático, el calentamiento global, la deforestación, la contaminación de los mares y la basura.

La discusión que surgió a partir de la presentación, generó mayores inquietudes. Aunque los estudiantes de sexto grado aceptaron la encomienda de participar del proyecto y desempeñarse como eco-ingenieros embajadores de la sustentabilidad, era evidente que tenían más preguntas que respuestas. Por lo tanto, se puede establecer que la situación motivadora cumplió con el criterio de emocionar, invitar y retar, pero sin frustrar el deseo de aprender.

\subsubsection{Clarificación del problema, proyecto y servicio}

El segundo paso de un currículo auténtico, de acuerdo con la estructura recomendada por Velázquez Rivera y Figarella García $(2016,2018)$, es la clarificación del problema, proyecto y servicio. Por medio de una nueva conversación los estudiantes mencionaron conocimientos previos y demostraron que ya estaban involucrados a nivel afectivo con la búsqueda de posibles soluciones al problema presentado. De esta manera, se continuó despertando la necesidad por aprender, facilitando que ocurran conexiones con experiencias previas. Los estudiantes se apoderaron del proceso de aprendizaje y de la necesidad de estudiar ecología. Como parte del proceso de clarificación, y para facilitar que se escuchara la voz de todos los estudiantes, cada uno completó la tabla KWH: What we Know?, What we Want to know? and $\underline{H}$ wow we want to learn? Luego, a base de las tablas individuales se creó una por grupo de trabajo, para finalmente construir, en sesión plenaria, una tabla que resumía la información de todo el grupo.

El análisis de las respuestas en la primera columna de la tabla y los argumentos presentados por los estudiantes en la discusión permitió a la maestra identificar posibles errores conceptuales que atendió más adelante al desarrollar las actividades de aprendizaje en la uni- 
dad. En la segunda columna, la mayoría de los temas mencionados por los estudiantes acerca de lo que querían aprender, coincidieron con los objetivos de aprendizaje sobre ecología que se habían planificado enseñar con anticipación. Las siguientes preguntas son algunos de los ejemplos de las necesidades de aprendizaje y los temas que los estudiantes querían aprender, incluyendo: ¿Cuáles son las condiciones que favorecen la vida en el planeta Tierra? ¿Qué debemos hacer para conservar la vida en los ecosistemas de la Tierra?

En la tercera columna de la tabla se facilitó la integración de actividades que ellos recomendaron realizar para aprender e investigar. Algunas de las actividades de aprendizaje sugeridas por los estudiantes fueron: buscar información y recursos de la Internet, invitar expertos para dar charlas, realizar viajes de campo, laboratorios y demostraciones.

Como resultado de este proceso, los estudiantes delimitaron el problema identificando algunos de sus componentes para los cuales redactaron nuevas preguntas que emergieron de la pregunta-problema principal. Estas preguntas, junto con el establecimiento de un orden en las actividades sugeridas para aprender (procedimiento a seguir) sirvieron de guía en el estudio del tema de ecología. La identificación de las actividades de aprendizaje (tercer paso) y el establecimiento del procedimiento a seguir por los estudiantes (cuarto paso) abonó a su autonomía y autodirección, los cuales son aspectos claves para facilitar el aprendizaje auténtico. Ambos constituyen el tercer y cuarto paso, respectivamente, en la estructura de un currículo centrado en la problematización en el aprendizaje.

\subsection{Actividades para aprender}

La implantación de las actividades de aprendizaje constituyen el quinto paso de la estructura de un currículo centrado en la problematización en el aprendizaje. Al comienzo de cada actividad desarrollada como parte de la unidad de ecología los estudiantes de sexto grado debían explicar por qué esa actividad fue identificada como importante y cómo les ayudaría a desempeñar su rol. Esta discusión continuó despertando en ellos la necesidad de aprender los temas específicos de cada lección. De esta forma, los temas relacionados con ecología que usualmente son estudiados en sexto grado en la clase de ciencias, ahora se aprendían en el contexto del proyecto, porque era de interés para los estudiantes. En términos generales, las actividades enriquecedoras desarrolladas a través del proyecto para facilitar la construcción de conocimientos, incluyeron: 1) inquirir continuo, 2) aprendizaje individual y grupal, 3) evaluación continua, 4) integración de la tecnología como herramienta para aprender y demostrar aprendizajes, e 5) integración de la comunidad escolar y de la comunidad experta externa. A continuación se describen algunas de las actividades medulares para aprender. 


\subsubsection{Charla con representante de UNA-USA PR}

La primera actividad identificada por los estudiantes como necesaria fue una charla de parte de quien les solicita su apoyo. El propósito de esta charla, era continuar conectando afectivamente a los estudiantes con el proyecto y con la necesidad de aprender de ecología. En esta charla participaron tanto los estudiantes de sexto grado como los futuros maestros de ciencias, quienes se apoyaban unos a otros en su proceso de aprender. Como parte de esta actividad, se destacó la importancia de los ODS y la necesidad de desempeñarse como ecoingenieros embajadores de la sustentabilidad. Era palpable la emoción de los estudiantes al sentirse necesarios e importantes para la UNA-USA PR. Durante la actividad expresaron su compromiso con el proyecto y su deseo de comenzar a aprender para desempeñar su rol.

\subsubsection{Reto por grupo de trabajo}

La maestra de ciencias le asignó a cada grupo de trabajo un reto que consistió en realizar una breve investigación que describiera las características e interacciones entre los factores bióticos y abióticos de los diferentes ecosistemas de Puerto Rico. Los ecosistemas asignados fueron: 1) Bosques, 2) Hidrografía, 3) Estuarios y 4) Arrecifes de Coral. El reto requirió que los estudiantes estudiaran el tema para responder a una serie de preguntas, las cuales guiaron la creación de una presentación digital. La misma, posteriormente fue compartida en un foro en línea con los futuros maestros de ciencias, con el fin de recibir su retrocomunicación. Una vez mejoradas las presentaciones digitales, los estudiantes de sexto grado compartieron su tema en la clase de ciencias para enseñar a sus compañeros acerca del ecosistema asignado.

\subsubsection{Foro en línea entre estudiantes y futuros maestros de ciencias}

La actividad del foro en línea consistió en el desarrollo de una comunicación asincrónica, utilizando una plataforma digital, para presentar el informe del reto (presentación digital) a los futuros maestros de ciencias. Los grupos de trabajo, estuvieron conformados por estudiantes de ambos niveles educativos (elemental y universitario) con el propósito de que los estudiantes de ambos grupos se conocieran e interactuaran. El foro en línea se desarrolló en dos actividades : 1) ¡A conocernos!- presentaciones de los integrantes de cada grupo compuesto por futuros maestros de ciencias y estudiantes de sexto grado con el fin de conocerse mejor, y 2) ¡A mejorar nuestros retos!- interacciones entre los integrantes de cada grupo para mejorar los informes del reto asignado. 
Los grupos de trabajo de sexto grado recibieron retrocomunicación, comentarios positivos y recomendaciones de parte de los futuros maestros de ciencias. Los mismos fueron discutidos en grupo para mejorar sus informes del reto y luego presentarlos en la clase. El propósito principal del foro en línea fue propiciar la interacción entre ambos grupos de estudiantes (sexto grado y futuros maestros de ciencias) de manera que unos aprendieran de otros, además de promover la autodirección en el aprendizaje de las ciencias y del desarrollo de destrezas tecnológicas.

\subsubsection{Viernes de STEM}

Durante tres meses, los viernes fueron dedicados a desarrollar actividades de integración de STEM, bajo el asesoramiento de expertos de STARBASE-PR. Este es un programa que surge de un consorcio entre la NASA y el Departamento de Defensa de Estados Unidos; entre sus propósitos está promover el aprendizaje de STEM en estudiantes escolares. En estos viernes también se integraron los futuros maestros de ciencias participantes del proyecto, quienes fueron responsables de dirigir algunas actividades de aprendizaje de los estudiantes de sexto grado. La mayoría de las veces, las actividades comenzaron con un reto que requirió que los estudiantes siguieran los pasos del diseño de ingeniería para resolverlo. Por medio de las actividades de STEM, los estudiantes de sexto grado aprendieron y se prepararon como ingenieros para posteriormente diseñar y construir el prototipo de biosfera. La creación del prototipo permitió a los estudiantes de sexto grado aplicar lo aprendido en torno a las condiciones necesarias para la vida. En varias ocasiones, el maestro de matemáticas se integró a estas actividades para explicar cómo resumir y representar conjuntos de datos numéricos relacionados con el tema particular que estaban trabajando.

\subsubsection{Laboratorios y demostraciones a base de inquirir}

La clase de ciencias se transformó en un verdadero lugar de exploración, manipulación y experimentación para los grupos de trabajo. Apoyados por las tecnologías, se desarrollaron actividades Hand's On-Minds On, que sirvieron de base para construir aprendizajes auténticos de ciencias acerca del tema de ecología. Entre ellas, los estudiantes destacaron la utilidad de las siguientes actividades: a) Laboratorios (El ciclo hidrológico, El efecto de la temperatura en los ecosistemas); b) Demostración (La contaminación de los suelos); c) Juegos (Rompecabezas ecológico y Cadenas/redes alimentarias). 


\subsubsection{Viaje de campo a ecosistemas de zona noreste de Puerto Rico}

Entre las actividades más impactantes para los estudiantes y que requirieron nuevamente la integración de los futuros maestros de ciencias, así como la comunidad escolar y personal experto, estuvieron los dos viajes de campo. El primer viaje de campo consistió en visitar la zona lluviosa en el noreste de PR. El mismo tuvo una duración de todo el día. Durante la mañana, se llevó a cabo un recorrido guiado por el estuario Río Espíritu Santo, por medio de una embarcación; mientras que en la tarde se realizó una caminata de exploración por veredas del Bosque Nacional del Caribe conocido como el Yunque.

Ambos recorridos le permitieron a los estudiantes descubrir las características distintivas de ecosistemas en la zona lluviosa de la isla y los tipos de interacciones que en ellos ocurren. De esta manera, tuvieron los elementos de comparación para luego estudiarlos con mayor profundidad y contrastarlos con ecosistemas equivalentes en zonas secas.

\subsubsection{Residencial Educativo en playa Buyé en Cabo Rojo}

El segundo viaje de campo fue parte del Residencial Educativo, el cual requirió pernoctar desde jueves en la mañana hasta el viernes en la tarde en la costa al suroeste de la isla, con el fin de estudiar los ecosistemas de esa zona seca y continuar desempeñando su rol como ecólogos y embajadores de la sustentabilidad. Algunas de las actividades que se realizaron fueron: a) charla en torno a las características de la zona, ofrecido por un ecólogo, b) recorrido guiado por el bosque seco y la zona costera del Refugio de Vida Silvestre de Cabo Rojo, y c) varias charlas educativas en torno a la historia del impacto humano en la zona, por ejemplo en las salinas y al hacer carbón.

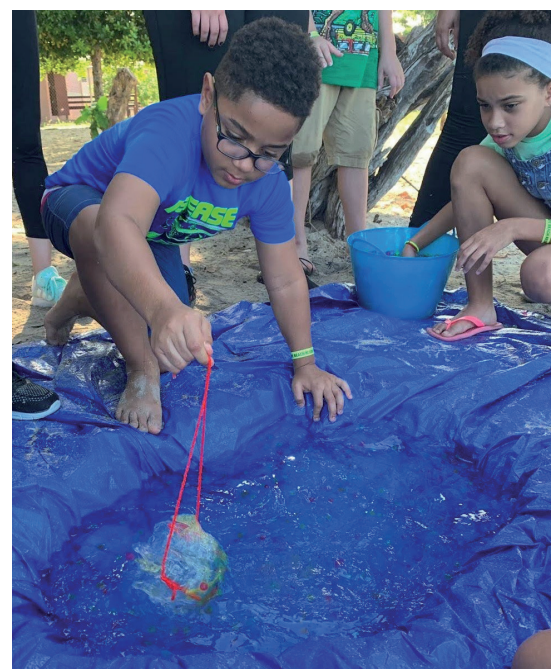

Foto 1. Estación de aprendizaje: Creación de un prototipo para recoger basura espacial integrando las disciplinas STEM y el diseño de ingeniería 
Una actividad del Residencial Educativo destacada fue la participación en las estaciones de aprendizaje creadas por futuros maestros de ciencias. En estas estaciones se integraron las disciplinas STEM y el diseño de ingeniería para la creación de prototipos que recreen la vida en el espacio o trabajen con el impacto humano en él. Los temas de las estaciones de aprendizaje fueron: a) Cómo nos protegernos en el espacio de la radiación solar, b) Cómo empacar alimentos para ser consumidos en el espacio y c) Cómo recoger basura espacial (ver Foto 1).

\subsubsection{Visita a la Sesión de la Asamblea Municipal de Cabo Rojo y recorrido por el Municipio}

El jueves en la tarde, como parte del Residencial Educativo, los estudiantes tuvieron la oportunidad de participar de una Sesión Legislativa de la Asamblea Municipal de Cabo Rojo con el fin de que vivieran procesos democráticos y políticos asociados con su rol como embajadores. Esto les permitió familiarizarse con procesos parlamentarios y de toma de decisiones propios en este tipo de reunión. Además, escucharon la presentación de proyectos para la conservación del ambiente que fueron aprobados en dicha sesión. Al finalizar la misma, los participantes del Proyecto Réplicas para la Vida, por medio de una resolución presentada y aprobada por los miembros de la Asamblea Municipal, fueron declarados "Huéspedes Distinguidos" del municipio. Luego, al llegar la noche, los estudiantes participaron de una charla educativa y de un recorrido guiado destacando aspectos culturales y la zona histórica del municipio de Cabo Rojo. Esta experiencia facilitó su posterior reflexión acerca de la relación entre el humano y su patrimonio cultural.

\subsection{Actividades para aplicar lo aprendido}

Las actividades para aprender sirvieron de fundamento para que los estudiantes aplicaran lo aprendido en la creación del producto solicitado. Según Velázquez Rivera y Figarella García (2016, 2018), el producto se refiere a las evidencias de ejecución que se utilizan para determinar el aprendizaje global o sumativo. El mismo requiere que el estudiante presente posibles soluciones al problema bajo estudio, así como el resultado del proyecto realizado y que ofrezca un servicio comunitario. Luego de creado el producto, este debe ser presentado públicamente, a la comunidad escolar, así como a la comunidad relacionada con el tema incluyendo a expertos, poblaciones impactadas y agencias o empresas.

La creación y presentación del producto son los últimos pasos (sexto y séptimo respectivamente) de un currículo auténtico centrado en la problematización en el aprendizaje. El 
producto, en el Proyecto Réplicas para la Vida, consistió de: a) recrear las condiciones necesarias para la vida al construir un prototipo de biosfera, b) presentar recomendaciones de cómo minimizar el impacto humano en los ecosistemas y conservar las condiciones necesarias para la vida, e c) implantar alguna de las recomendaciones sugeridas.

\subsubsection{Creación del prototipo de biosfera}

La creación del prototipo de biosfera, facilitó que los estudiantes lograran observar y estudiar las condiciones necesarias para la vida. Además, les permitió comprender, en su rol como ecoingenieros, las interacciones importantes que ocurren entre los factores bióticos y abióticos de un ecosistema. Algunos de los materiales y animales utilizados para construir el prototipo de biosfera incluyeron: suelo, plantas, agua, insectos, gusanos y materiales reciclables. El diseño de ingeniería y la integración de las disciplinas STEM fueron medulares en la creación grupal de los prototipos de biosfera. Al cabo de dos semanas, los estudiantes aplicaron nuevamente lo aprendido al analizar los resultados de lo ocurrido en los prototipos de biosferas (ver Foto 2). En las reflexiones discutidas con los estudiantes argumentaron lo difícil que es replicar las condiciones de un ecosistema para que los organismos permanecieran con vida y lo importante que es conocer el funcionamiento de los ciclos biogeoquímicos de la naturaleza.

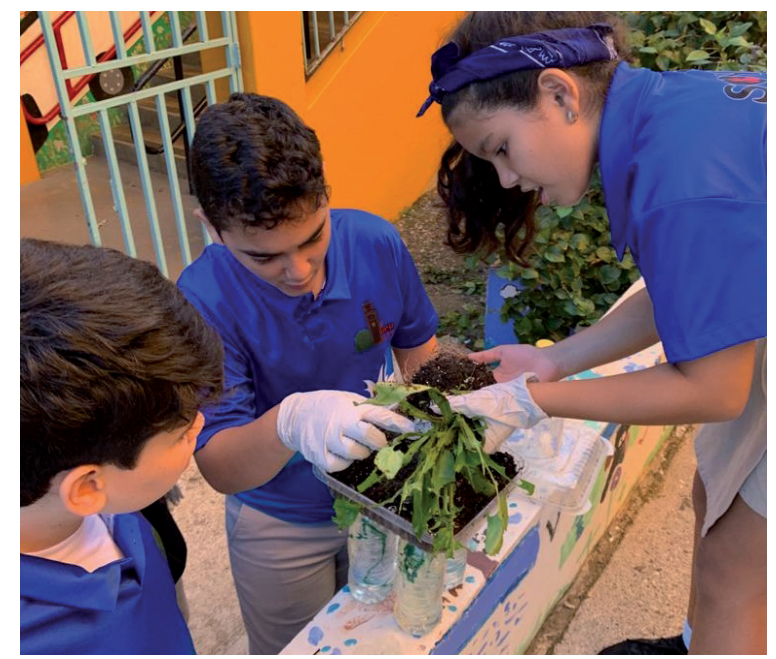

Foto 2. Análisis y resultados del prototipo de biosfera en el cual se recrearon las condiciones necesarias para la vida

\subsubsection{Actividad del clausura del Proyecto}

La actividad de Clausura del Proyecto Réplicas para la Vida fue un momento de celebración de los logros alcanzados y del trabajo llevado a cabo apoyado por distintos sectores de la 
comunidad. En el mismo estuvieron presentes autoridades de la escuela y de la universidad, familiares y representantes de la comunidad experta (UNA-USA PR y STARBASE), además de los estudiantes de sexto grado y de los futuros maestros de ciencias, acompañados por sus respectivos maestros. En esta actividad, los estudiantes compartieron el proceso de aprendizaje vivido, presentaron los prototipos de biosfera creados, así como su reflexión para mejorarlos. Esto seguido de los datos recopilados en torno al impacto a los ecosistemas visitados, las recomendaciones para minimizar este impacto, y su plan para implantar una de ellas. Concluida la presentación de los estudiantes, algunos miembros de la comunidad, comenzando con los expertos, ofrecieron su retrocomunicación al trabajo realizado. Esta dinámica le permitió a los estudiantes presentar y defender su producto, además de formularse nuevas interrogantes en torno al problema estudiado en el proyecto. La actividad culminó con la entrega de certificados para todos los participantes del proyecto.

\subsubsection{Prestación del servicio a la comunidad: Campaña educativa}

Los estudiantes de sexto grado identificaron un problema de basura en su escuela. Se percataron que una de las fuentes de basura eran los vasos desechables que utilizaban para tomar agua en cada salón. Por tal razón, decidieron atender esta problemática presentando una propuesta para minimizar esa fuente de desperdicios generada por ellos. Mediante el apoyo de la asociación de padres de la escuela, llevaron a cabo una campaña educativa en su comunidad para concienciar en torno a cómo reducir la cantidad de basura y el uso efectivo de los vasos desechables. Esta propuesta incluyó que cada estudiante tuviera acceso a un envase reusable (termo insulado) para servirse agua en la escuela.

\section{5. ¿CUÁLES FUERON LOS LOGROS DEL PROYECTO?}

A continuación se presentan los logros más importantes del Proyecto identificados en una evaluación colaborativa que se llevó a cabo.

Los logros de la problematización en el aprendizaje de los estudiantes, por medio del Proyecto Réplicas para la Vida, fueron documentados. En la Tabla 1, se presentan los logros más importantes identificados según la estructura y las actividades medulares del proyecto. 
Tabla 1. Logros de la problematización en el aprendizaje de los estudiantes según la estructura y las actividades medulares del proyecto

\begin{tabular}{lll}
\hline ESTRUCTURA DEL & ACTIVIDADES & LOGROS DE LA PROBLEMATIZACIÓN EN EL \\
PROYECTO & MEDULARES & APRENDIZAJE DE LOS ESTUDIANTES \\
\hline
\end{tabular}

Actividades para facilitar Presentación del problema, la problematización del rol y del producto solicitado

Clarificación del problema, proyecto y servicio

\begin{tabular}{|c|c|c|}
\hline & $\begin{array}{l}\text { Clarificación del problema, } \\
\text { proyecto y servicio }\end{array}$ & $\begin{array}{l}\text { Descubrieron conocimiento previo y cómo lo aprendieron } \\
\text { (metacognición), así como sus necesidades de aprendiza- } \\
\text { je; de las cuales algunas de ellas fueron identificadas por la } \\
\text { maestra como errores conceptuales. Además, identificaron y } \\
\text { establecieron actividades de aprendizaje que se debían desa- } \\
\text { rrollar (la opinión de los estudiantes fue considerada en todo } \\
\text { momento para realizar los ajustes en las actividades y en los } \\
\text { recursos, con el fin de promover la autodirección de su proce- } \\
\text { so de aprendizaje). }\end{array}$ \\
\hline \multirow[t]{3}{*}{$\begin{array}{l}\text { Actividades } \\
\text { para aprender }\end{array}$} & $\begin{array}{l}\text { Charla con representante de } \\
\text { UNA-USA PR }\end{array}$ & $\begin{array}{l}\text { Reconocieron la pertinencia y relevancia del problema acerca } \\
\text { del impacto humano. De igual manera, reconocieron la nece- } \\
\text { sidad de aprender de ecología para conservar las condiciones } \\
\text { de vida de la Tierra de manera que no sea necesario replicar- } \\
\text { las. Además, se familiarizaron con los ODS y valoraron la im- } \\
\text { portancia de asumir un rol activo para alcanzarlos y presentar } \\
\text { soluciones alternativas al problema presentado. }\end{array}$ \\
\hline & Reto por grupo de trabajo & $\begin{array}{l}\text { Desarrollaron competencias tecnológicas al identificar y eva- } \\
\text { luar fuentes apropiadas de información en línea. Luego, anali- } \\
\text { zaron y comunicaron sus hallazgos al redactar un informe. El } \\
\text { mismo fue presentado por medio de una presentación digital } \\
\text { que crearon, en la cual se integraron texto, imágenes, videos } \\
\text { y referencias. }\end{array}$ \\
\hline & $\begin{array}{l}\text { Foro en línea entre } \\
\text { estudiantes y futuros } \\
\text { maestros de ciencias }\end{array}$ & $\begin{array}{l}\text { Desarrollaron destrezas de comunicación escrita y tecnológi- } \\
\text { ca. Además, participaron eficazmente en una serie de discu- } \\
\text { siones colaborativas (uno a uno, en grupos y dirigidos por las } \\
\text { maestras) acerca del contenido de ciencias y problemáticas, } \\
\text { basándose en las ideas de los demás y expresando las pro- } \\
\text { pias con claridad. }\end{array}$ \\
\hline
\end{tabular}

Se despertó la necesidad de aprender de ecología y de desempeñar un rol activo por el bien de la sociedad, al investigar un problema actual y utilizar lo que se va a aprender para colaborar con una agencia o entidad real que necesita de ellos. 


\begin{tabular}{|c|c|}
\hline Viernes de STEM & $\begin{array}{l}\text { Aprendieron conceptos relacionados con las disciplinas } \\
\text { STEM, a la vez que utilizaron el diseño de ingeniería al desa- } \\
\text { rrollar soluciones para retos presentados en las actividades. } \\
\text { La integración de las disciplinas STEM y del diseño de inge- } \\
\text { niería permitió el diseño de un prototipo de biosfera sellado } \\
\text { en el cual se debían replicar las condiciones necesarias para } \\
\text { la vida. }\end{array}$ \\
\hline $\begin{array}{l}\text { Laboratorios y } \\
\text { demostraciones } \\
\text { a base de Inquirir }\end{array}$ & $\begin{array}{l}\text { Conectaron conceptos científicos al participar de experien- } \\
\text { cias prácticas que retaron el conocimiento previo. Además, } \\
\text { trabajaron con modelos y analizaron e interpretaron datos } \\
\text { para contestar preguntas relacionadas con el conflicto ge- } \\
\text { nerado. Esto requirió obtener, evaluar y comunicar continua- } \\
\text { mente información científica relacionada con el problema } \\
\text { bajo estudio. }\end{array}$ \\
\hline $\begin{array}{l}\text { Viaje de campo a ecosistemas } \\
\text { de Zona noreste de PR (zona } \\
\text { lluviosa) Estuario Río Espíritu } \\
\text { Santo El Yunque }\end{array}$ & $\begin{array}{l}\text { Apoyados por expertos, los estudiantes describieron y com- } \\
\text { pararon características e interacciones entre los factores bió- } \\
\text { ticos y abióticos de los ecosistemas visitados (estuarinos y el } \\
\text { bosque tropical). Infirieron características del río que da ori- } \\
\text { gen al estuario y del ecosistema marino que se observó a la } \\
\text { distancia desde el bosque. Además, hicieron predicciones de } \\
\text { cómo serían esas características en el suroeste de PR. Esto los } \\
\text { llevó a estudiar en clase las interacciones entre los factores } \\
\text { bióticos y abióticos. }\end{array}$ \\
\hline $\begin{array}{l}\text { Residencial Educativo } \\
\text { en playa Buyé en Cabo Rojo }\end{array}$ & $\begin{array}{l}\text { Aprendieron el contenido de STEM y aplicaron el diseño de } \\
\text { ingeniería al intentar replicar las condiciones de vida en el } \\
\text { espacio en las estaciones de aprendizaje. Estas fueron: 1. Im- } \\
\text { pacto a la atmósfera: ¿Cómo recolectar la basura espacial?, } 2 . \\
\text { Vida en el espacio: ¿Cómo empacar alimentos? y 3. Vida en el } \\
\text { espacio: ¿Cómo protegernos de la radiación solar? Además, } \\
\text { indagaron en los conceptos ecológicos bajo estudio, apli- } \\
\text { caron destrezas científicas (observar, inferir, predecir, entre } \\
\text { otras), intercambiaron ideas de manera oral y escrita (comu- } \\
\text { nicación) y aprendieron científicamente a cómo aprender. }\end{array}$ \\
\hline
\end{tabular}




\begin{tabular}{|c|c|c|}
\hline & $\begin{array}{l}\text { Visita a la Sesión de la Asam- } \\
\text { blea Municipal de Cabo Rojo } \\
\text { y recorrido por el Municipio }\end{array}$ & $\begin{array}{l}\text { Aprendieron cómo se presenta y defiende los proyectos de } \\
\text { sustentabilidad ambiental ante el gobierno, ayudándoles a } \\
\text { tener mejor entendimiento de los asuntos ambientales que } \\
\text { afectan a las sociedades contemporáneas. } \\
\text { Identificaron relaciones entre momentos históricos y el im- } \\
\text { pacto humano en el medio ambiente. } \\
\text { Reconocieron la importancia de asumir un rol activo en la so- } \\
\text { ciedad. Para los estudiantes esto se validó cuando el Munici- } \\
\text { pio Autónomo de Cabo Rojo y su Legislatura Municipal los de- } \\
\text { claró "Huéspedes distinguidos" (por medio de la Resolución } \\
\text { \# 17, del Proyecto \#26 de la Sesión ordinaria del 2019-2020). }\end{array}$ \\
\hline \multirow[t]{3}{*}{$\begin{array}{l}\text { Actividades para aplicar } \\
\text { lo aprendido }\end{array}$} & $\begin{array}{l}\text { Creación del prototipo de } \\
\text { biosfera }\end{array}$ & $\begin{array}{l}\text { Demostraron comprensión de conceptos de las ciencias } \\
\text { como son: los ciclos biogeoquímicos, las interacciones entre } \\
\text { los organismos y su ambiente, y las relaciones simbióticas } \\
\text { entre los organismos. Evidenciaron comprensión y consisten- } \\
\text { cia en el uso de los conocimientos que construyeron acerca } \\
\text { de ecología y de STEM al revisar sus diseños, construir los } \\
\text { prototipos y proponer soluciones. }\end{array}$ \\
\hline & $\begin{array}{l}\text { Actividad de clausura del pro- } \\
\text { yecto }\end{array}$ & $\begin{array}{l}\text { Celebraron sus logros de aprendizaje y reconocieron la im- } \\
\text { portancia del conocimiento científico construido para poder } \\
\text { modificar sus acciones y las de los demás con el fin de reducir } \\
\text { un problema que es significativo y relevante a nivel local y } \\
\text { mundial. }\end{array}$ \\
\hline & $\begin{array}{l}\text { Prestación del servicio a la } \\
\text { comunidad: Campaña educa- } \\
\text { tiva }\end{array}$ & $\begin{array}{l}\text { Implantaron recomendaciones para propiciar la sustentabili- } \\
\text { dad; en estas se incluyeron recomendaciones de cambios en } \\
\text { sus conductas (reconociendo su responsabilidad ciudadana), } \\
\text { así como de cambios necesarios en la escuela, la comunidad } \\
\text { y el país. }\end{array}$ \\
\hline
\end{tabular}

Además, se realizó un grupo focal con el propósito de conocer la experiencia y los aprendizajes de los estudiantes durante el proyecto. Desde la perspectiva de los estudiantes, estas experiencias educativas en contextos reales les ayudó a aprender los contenidos curriculares relacionados con ecología. De igual manera, este proyecto les permitió experimentar aprendizajes significativos y construir nuevos conocimientos para aplicarlos en el mundo real, tanto a nivel personal como académicamente. Además, se visualizaron a sí mismos como agentes de cambio positivo en la sociedad por medio de su rol. Específicamente, un estudiante indicó que:

“... (el proyecto) me permitió asumir el rol de eco-ingeniero embajador de la sustentabilidad enseñando a otros niños y personas que protegan (protejan) la naturaleza cuidándola, conservándola y reconociendo su importancia." 


\subsection{Otros logros importantes del Proyecto}

Otro de los logros más importantes del proyecto fue el reconocimiento que recibieron los estudiantes como "Ciudadanos Distinguidos" de Cabo Rojo otorgado por la Legislatura Municipal y el mensaje de agradecimiento recibido por el representante de la UNA-USA PR en la actividad de clausura. Para los estudiantes fue muy importante que estas entidades de PR reconocieran su labor en la construcción y aplicación del conocimiento científico a la solución de los problemas ambientales. Por otra parte, un logro para las maestras involucradas en el diseño del Proyecto Réplicas para la Vida, fue la forma en que se atendieron los retos de reducir costos y coordinar las actividades. Por tal razón, la colaboración y la planificación conjunta permitió sincronizar recursos de la escuela, los padres, la comunidad científica y del gobierno para lograr desarrollar el proyecto.

\section{CONCLUSIÓN}

El estudio del impacto humano en los ecosistemas del planeta Tierra, junto con el reto de desempeñar el rol de eco-ingenieros embajadores de la sustentabilidad, sirvió de contexto para que estudiantes de sexto grado aprendieran de manera auténtica acerca de ecología. Por medio del Proyecto Réplicas para la Vida, se despertó la necesidad de aprender de ecología, al tener que replicar las condiciones necesarias para la vida integrando las disciplinas STEM y el diseño de ingeniería en la creación de un prototipo de biosfera.

Las actividades para facilitar la problematización, para aprender y para aplicar lo aprendido promovieron el desarrollo de aprendizajes auténticos, pertinentes y valorados por los estudiantes. Por lo tanto, para que el aprendizaje de las ciencias sea auténtico debe ser valorado por los estudiantes como algo primordial, y la experiencia de aprendizaje debe ser útil y aplicable al mundo real en el que viven. Por tal razón, se concluye que la problematización es una herramienta útil para facilitar el aprendizaje auténtico de las ciencias en el nivel elemental.

\section{REFERENCIAS BIBLIOGRÁFICAS}

Figarella García, F. (2018). De “iescucha!, ¡copia! y jrepite! a ¡investiga!, jcomparte! y jcrea!”: El enfoque constructivista y las interacciones en la sala de clases. CoopERA. 
Naciones Unidas. (2020). Objetivos de desarrollo sostenible. https://www.un.org/sustainabledevelopment/es/sustainable-development-goals/

Newmann, F., Marks, H., \& Gamoran, A. (1995, April 18-22). Authentic pedagogy and students performance [Paper presentation]. American Educational Research Association Annual Meeting, San Francisco, CA, United States.

Piaget, J. (1980). Psicología y pedagogía. Ariel.

Velázquez Rivera, L. M. y Figarella García, F. (2018). La problematización en el aprendizaje: Tres estrategias para la creación de un currículo auténtico. CoopERA.

Velázquez Rivera, L. M., Figarella García, F. y Clark Mora, L. (2016). La aventura del currículo auténtico: Posibilidades y éxitos en la problematización del aprendizaje. CoopERA. 